\title{
Swimming Speed of The Breaststroke Kick
}

\author{
by \\ Marek Strzała1, Piotr Krężałek², Marcin Kaca³, Grzegorz Głąb4, Andrzej Ostrowski ${ }^{5}$, \\ Arkadiusz Stanula ${ }^{6}$, Aleksander Tyka
}

The breaststroke kick is responsible for a considerable portion of the forward propulsion in breaststroke swimming. The aim of this study was to measure selected anthropometric variables and functional properties of a swimmer's body: length of body parts; functional range of motion in the leg joints and anaerobic power of the lower limbs. Chosen kinematic variables useful in the evaluation of swimming performance in the breaststroke kick were evaluated. In the present research, swimming speed using breaststroke kicks depended to the largest extent on anaerobic endurance $(0.46, p<0.05$ partial correlations with age control). In addition, knee external rotation and swimming technique index had an impact on swimming speed and kick length (both partial correlations with age control 0.35, $p<$ 0.08). A kinematic analysis of the breaststroke kick hip displacement compatible with horizontal body displacement was significantly negatively correlated with foot slip in the water opposite to body displacement (partial correlations: with leg length control $-0.43, p<0.05$; with shank length control $-0.45, p<0.05$, respectively). Present research and measurements of selected body properties, physical endurance and kinematic movement analysis may help in making a precise determination of an athlete's talent for breaststroke swimming.

Key words: swimming, breaststroke kick, kinematic analysis

\section{Introduction}

In breaststroke swimming, according to the International Federation for Swimming FINA (Fédération Internationale de Natation Amateur), rules that do not apply to any other swimming style determine the stroke cycle from the start and throughout the race; the stroke cycle must consist of an arm stroke and a leg kick that occur in sequence. During each complete cycle, the arm stroke and the leg kick produce similar propulsion forces. In tethered swimming, for example, the peak and mean values of tether forces measured during the propulsion caused by the upper and lower extremities were similar (Yeater et al., 1980).
Early research on advanced kinematic analysis proved that the leg kick is the largest propulsive force of the stroke and that it occurs as the third propulsive force in the breaststroke movement cycle (Mason et al., 1989). The first of the forces, the 'catch', was associated with the hand pull. The second propulsive stage was hypothesised to be the swimmer catching the wave produced by the swimming action itself with the relatively large surface of the trunk moving forwards against a still, incompressible water volume (Mason et al., 1989). It was also recognised in a study by Kippenhan (1991) that the breaststroke kick, especially the whip kick,

1 - Department of Theory and Methodology of Water Sports, University School of Physical Education, Cracow, Poland.

2 - Department of Physiotherapy Faculty of Motor Rehabilitation University School of Physical Education, Cracow.

3 - Department of Theory and Methodology of Water Sports, University School of Physical Education, Cracow, Poland.

4 - Department of Physiotherapy Faculty of Motor Rehabilitation University School of Physical Education, Cracow, Poland.

5 - Department of Theory and Methodology of Water Sports, University School of Physical Education, Cracow, Poland.

6 - Department of Sports Theory, The Jerzy Kukuczka Academy of Physical Education, Katowice, Poland.

7 - Department of Physiology and Biochemistry, University School of Physical Education, Cracow, Poland. 
makes a major contribution to the forward propulsion in this style.

Moreover, Leblanc et al. (2007) observed that in elite swimmers, a higher peak forward velocity was reached during arm propulsive phases when swimming distances of 50 and $100 \mathrm{~m}$ at race-pace. Maglischo (2003) explained that swimmers accelerated their bodies for a longer time with their arms than they did with their legs, but the kick is clearly a dominant propulsive force in the breaststroke. Propulsion from the kick begins when the forward velocity is the lowest in the stroke cycle, whereas the legs begin to accelerate the body forwards together with the arms when they are already traveling much faster. This occurs at the highest breaststroke velocities.

A correctly performed whip kick is challenging to execute. Difficulties are generally associated with complicated hip, knee, and ankle joint motion sequences (Kippenhan, 1991). A high range of motion, flexibility and above-average musculoskeletal forces during the movement produce powerful whip kick propulsion. The structure of one's legs and feet may also play a role in becoming a good breaststroker, described by others as "walking like a duck" (Bixler, 2005). These characteristics are visible to the coach and may be measured. Indeed, in a study by Kippenhan (2002), it was noted that in a group of collegiate varsity and recreational swimmers, the more skilled ones had a larger external knee rotation. In an investigation conducted by Jagomägi and Jürimäe (2005) on a large sample of 125 female swimmers, the swimming velocity in a $100 \mathrm{~m}$ breaststroke performed using a kickboard and legs was significantly influenced by the flexibility of the hip, knee and ankle. This swimming velocity was also significantly related to the body height and body mass and to the standing broad jump. Gerard et al. (1986) showed a statistically significant direct dependence between anaerobic power measured by vertical jump results and type II fibre content in the vastus lateralis muscle with a higher efficiency in shortrather than long-distance swimming.

Considering these data, we measured several anthropometric indices and functional properties of the breaststroke: length of body parts, range of motion in the leg joints and anaerobic power of the lower limbs. We also constructed kinematic indices useful in the evaluation of swimming performance of the breaststroke kick. We hypothesised that the above-mentioned indices may influence the basic swimming technique variables: stroke rate and stroke length of the breaststroke kick and swimming speed using a kickboard and legs only.

\section{Methods}

\section{Participants}

Volunteers were recruited from two sport schools and from a university swimming club. They or their parents signed a written informed consent before taking part in the research, which was approved by the Bioethics Committee in Cracow. The 27 swimmers representing regional and national sports levels trained twice a day, six times a week. The swimmers were $15.7 \pm 1.98$ years old; 26 were between 14 to 19 years old, and one was 21 years old. The average body height was $180.1 \pm 7.21 \mathrm{~cm}$, and average body mass was $70.3 \pm 9.35 \mathrm{~kg}$. The subjects specialised in the breaststroke and in the individual medley.

\section{Anthropometry}

The anthropometric data that was gathered made it possible to determine the swimmers' somatotypes according to Carter-Heath (1990). Within the sample, 18 swimmers represented the mesomorphic somatotype, and 9, the ectomorphic one. The measurements were conducted with appropriate anthropometric apparatus (Sieber Hegner Maschinen AG, Switzerland; the Harpenden-type skinfold callipers with a constant pressure of $10 \mathrm{~g} / \mathrm{cm}^{-2}$ were used). The percentage of body fat was $10.21 \pm 2.41 \%$. Lower extremity and shank lengths (Lg-L and Sh-L, respectively) were measured in a recumbent position from the trochanter major and head of the fibula, respectively, to the toes with full plantar flexion.

\section{Leg joint mobility}

The range of motion for each joint in the lower extremity was measured, preceded by a warm-up similar to that used in competition conditions without specific stretching, using a plastic goniometer by an experienced physiotherapist. A reference angle of $0 \stackrel{\circ}{\circ}$ was assumed for the sagittal plane orientation. Hip joint internal rotation (Hip-Int) was measured in the sitting position by bending to a maximal bending angle close to $90 \stackrel{\circ}{\circ}$. Knee external rotation (Knee-Ext) was measured in a horizontal position lying prone by bending the joint to an angle close 


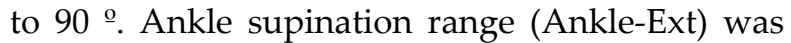
also measured in the same position, but with mechanical knee and shank immobilisation. The measurement reliability was high; the range was $1^{\mathrm{o}}$ for repeated measurements. The mean value of the range of motion for the right and left hip joints was registered. The same procedure was conducted for knees and ankles.

\section{Anaerobic endurance}

Maximal power and work output during vertical jumps performed in a 15-second period $(15 \mathrm{~s}-\mathrm{VJ})$ were recorded using the Opto Jump (Italy) apparatus. The height of the centre of gravity during the vertical jump (CMJ) was assessed using the jump duration formula 1 . Work during the jump (W) was calculated according to formula 2 .

$$
\begin{gathered}
\Delta h=\frac{g t_{l}^{2}}{8} \\
W=m g \Delta h=\frac{m g^{2} t_{l}^{2}}{8}
\end{gathered}
$$

where: $W L$ - work during the $m$-body mass jump [J]

$g$ - gravitational acceleration

$t_{l}$ - jump duration

$\left[\mathrm{m} \mathrm{s}^{-2}\right]$

[s]

$h$ - elevation of the centre of

gravity $[m]$

The $50 \mathrm{~m}$ all-out swimming speed tests were carried out in a 25 meter-long swimming pool, with the start in the water using a kickboard and breaststroke kick only.

All swimmers used the same standard kickboard, which was positioned against the upper part of the body identically for every swimmer. The so-called high grip of the kickboard was used.

\section{Underwater recordings}

The swimmers' body movements were recorded using a Canon Legria HV40 (Japan) camcorder and an underwater camera, a Sony Color Submergible Camera IP:68 (Japan). Video recordings were made with the frequency of 50 frames per second. The recording took place from the side with the camcorder installed on a portable trolley moving along the swimming pool border parallel to the swimmer. A person trained to operate the above-mentioned device pulled the trolley in both directions along the swimming pool in such a way as to not to cross the perpendicular lines between the swimmer's head and the lens at the front and the toes of the stretched legs at the back. The underwater camera was mounted to the lower arm of the trolley and emerged in water approximately 1 meter below the surface at a distance of approximately 5 meters from the swimmer's lane.

\section{Basic video analysis of breaststroke kick swimming}

The video analysis made it possible to determine the moments in which characteristic events of the swimming cycle occurred, i.e., the start of propulsive movement of the lower extremities at the beginning of the backward leg movement at the moment the stretching began in the knee joint.

The video analysis made it possible to identify the characteristic cyclic elements of leg movements in the breaststroke kick: kick rate (KR) and kick length (KL), which was calculated as the ratio between $\mathrm{KR}$ and the average swimming speed (V) for the $50 \mathrm{~m}$ distance.

The duration of the race $\Delta t_{i}$ and times within separate sectors were measured with a stopwatch with accuracy of $0.01 \mathrm{~s}$.

The following parameters were used to assess swimming technique during each analysed $25 \mathrm{~m}$ swim $(\mathrm{i}=1,2)$ :

1) Swimming speed: $V_{i}=25 \mathrm{~m} / \Delta \mathrm{t}_{\mathrm{i}}\left[\mathrm{m} \cdot \mathrm{s}^{-1}\right]$,

2) Kick rate $\left(\mathrm{KR}_{\mathrm{i}}\right)$, calculated as the reciprocal of the arithmetical average of the duration of three analysed swimming cycles: $K R_{i}=1 / T_{i}$ [ckl·min-1],

3) Kick length $\left(\mathrm{KLi}_{\mathrm{i}}\right)$, calculated as the average speed over $K R_{i}$ ratio: $K L_{i}=V_{i} / K R_{i}[m]$.

The results of swimming technique variables $K R$ and $K L$ and the kinematic indices described below are presented as the means calculated from six full leg movement cycles, from which 3 were identified in the $5-20 \mathrm{~m}$ sector and the other 3 were identified in the $30-40 \mathrm{~m}$ sector of the $50 \mathrm{~m}$ distance.

\section{Kinematic analysis of the breaststroke kick}

The analysis of the ankle and hip movement was performed using KA Video software - R. Schleihauf, San Francisco State University (2006). At first, a scaling coefficient for every athlete was obtained using anthropometric measurements. Then, the positions of markers located on ropes on both sides of the lane and the positions of the hip and ankle joints were identified in every recorded frame. A global 
reference system was selected with the origin at the midpoint between lane rope markers. Afterwards, global coordinates of the hip and ankle joints were calculated for every film frame. The phase-ending event, when the distance between the hip and ankle is maximal, was identified for every movement cycle. The relative displacements of the ankle and hip joints $\mathrm{d}_{\mathrm{A}-\mathrm{H}}[\mathrm{m}]$ and the displacements of these joints in the global reference system $\left(\mathrm{d}_{\mathrm{A}}[\mathrm{m}]\right.$ - feet slip, $\mathrm{d}_{\mathrm{H}}[\mathrm{m}]$ - body forward movement) during the whole phase were calculated. Finally, quotients were calculated for the ankle and hip displacements and their relative displacements $\mathrm{d}_{\mathrm{rA}}=\mathrm{d}_{\mathrm{A}} / \mathrm{d}_{\mathrm{A}-\mathrm{H}}[\%]$ and $\mathrm{d}_{\mathrm{rH}}=\mathrm{d}_{\mathrm{H}} / \mathrm{d}_{\mathrm{A}-\mathrm{H}}$ [\%].

\section{Statistical methods}

Means and standard deviations were calculated. To determine the relationship between swimming speed with somatic, functional, swimming technique variables and anaerobic capacity indices, the partial correlations, controlling for age, were computed. The relationships between kinematic indices (Hip displacement and Ankle displacement) were also partial correlations computed by controlling for leg (Lg-L) and shank length (Sh-L), knee rotation and anaerobic capacity indices. The Pearson correlation coefficient was computed between KL and lower extremity indices Lg-L and Sh-L. A normal distribution of data was registered. All tests were conducted with STATISTICA 6.1 software (StatSoft, Inc). The significance level was set at $p<0.05$.

\section{Results}

Mean swimming speed using the breaststroke kicks for a distance of $50 \mathrm{~m}$ (V50) was $1.10 \pm 0.06 \mathrm{~m} \cdot \mathrm{s}^{-1}$. The partial correlation measurements with age control between somatic and functional variables are shown in Table 1 . The anaerobic endurance index showed an average relation with swimming speed.

Partial correlations on the verge of statistical significance between the KL index and the knee joint movement range were noted (Table 2). The KL correlation, without age normalisation, with lower extremity length indices Lg-L and Sh$\mathrm{L}$ showed a dependence on the verge of statistical significance of $0.34, \mathrm{p}=0.08$, and $0.37, \mathrm{p}<0.06$, respectively.

A dependence on the verge of statistical significance was reached between the knee joint external rotation range and swimming speed with age control (Table 2).

Kinematic indices of the propulsion phase of breaststroke kicks with age control in most cases significantly influenced V50 values and SL values (Table 3 ).

The KL index of the breaststroke kick technique analysis did not show other significant correlations.

Significantly greater hip displacement in the propulsive movement was negatively correlated with foot slip in the water with lower extremity length indices control, Lg-L and Sh-L (Table 4).

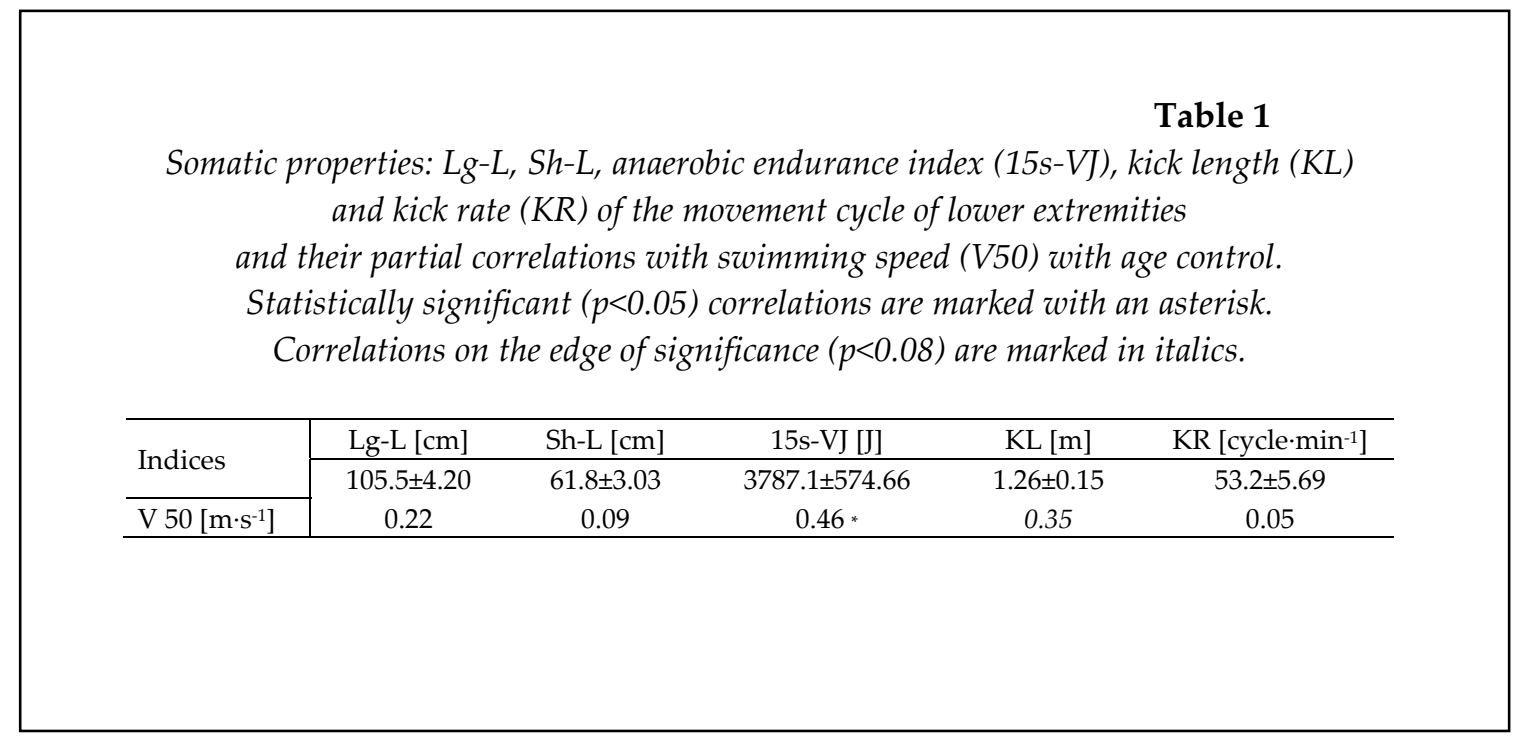




\begin{tabular}{|c|c|c|c|}
\hline \multicolumn{4}{|c|}{$\begin{array}{c}\text { Table } 2 \\
\text { Hip, knee and ankle joint movement range in the lateral plane and their partial correlations } \\
\text { with swimming speed }(V 50) \text { with age control. } \\
\text { Correlations on the edge of significance }(p<0.08) \text { are marked in italics. }\end{array}$} \\
\hline \multirow{2}{*}{ Indices } & Hip-Int [ํ] & Knee-Ext [ํ] & Ankle-Ext [ํ] \\
\hline & $35.3 \pm 7.25$ & $44.0 \pm 8.48$ & $30.6 \pm 7.06$ \\
\hline $\mathrm{V} 50\left[\mathrm{~m} \cdot \mathrm{s}^{-1}\right]$ & 0.08 & 0.35 & 0.09 \\
\hline
\end{tabular}

\begin{tabular}{|c|c|c|c|c|c|c|}
\hline \multicolumn{7}{|c|}{$\begin{array}{c}\text { Table } 3 \\
\text { Kinematic indices of the breaststroke kick propulsion phase and their partial correlations } \\
\text { with swimming speed (V50) and leg movement cycle length }(K L) \text { with age control. } \\
\text { Statistically significant correlations are marked as follows: } p<0.05^{*}, p<0.01^{* *} \text {. }\end{array}$} \\
\hline \multirow[t]{2}{*}{ Indices } & $\mathrm{dH}_{\mathrm{H}}[\mathrm{m}]$ & $\mathrm{d}_{\mathrm{A}}[\mathrm{m}]$ & $\mathrm{d}_{\mathrm{rH}}[\%]$ & $\mathrm{drA}_{\mathrm{rA}}[\%]$ & $\begin{array}{c}\mathrm{VH}_{\max } \\
{\left[\mathrm{m} \cdot \mathrm{s}^{-1}\right]}\end{array}$ & $\begin{array}{l}\text { VHaver } \\
{\left[\mathrm{m} \cdot \mathrm{s}^{-1}\right]}\end{array}$ \\
\hline & $0.32 \pm 0.03$ & $0.29 \pm 0.04$ & $0.52 \pm 0.05$ & $0.48 \pm 0.05$ & $1.54 \pm 0.11$ & $1.16 \pm 0.08$ \\
\hline $\begin{array}{l}\text { V } 50\left[\mathrm{~m} \cdot \mathrm{s}^{-1}\right] \\
\mathrm{KL}[\mathrm{m}]\end{array}$ & $\begin{array}{l}0.67^{* *} \\
0.63^{* *}\end{array}$ & $\begin{array}{l}-0.19 \\
-0.23\end{array}$ & $\begin{array}{l}0.46 \text { * } \\
0.46 *\end{array}$ & $\begin{array}{l}-0.46 \text { * } \\
-0.46 \text { * }\end{array}$ & $\begin{array}{c}0.58^{* *} \\
0.21\end{array}$ & $\begin{array}{l}0.78 \text { ** } \\
0.39 *\end{array}$ \\
\hline
\end{tabular}

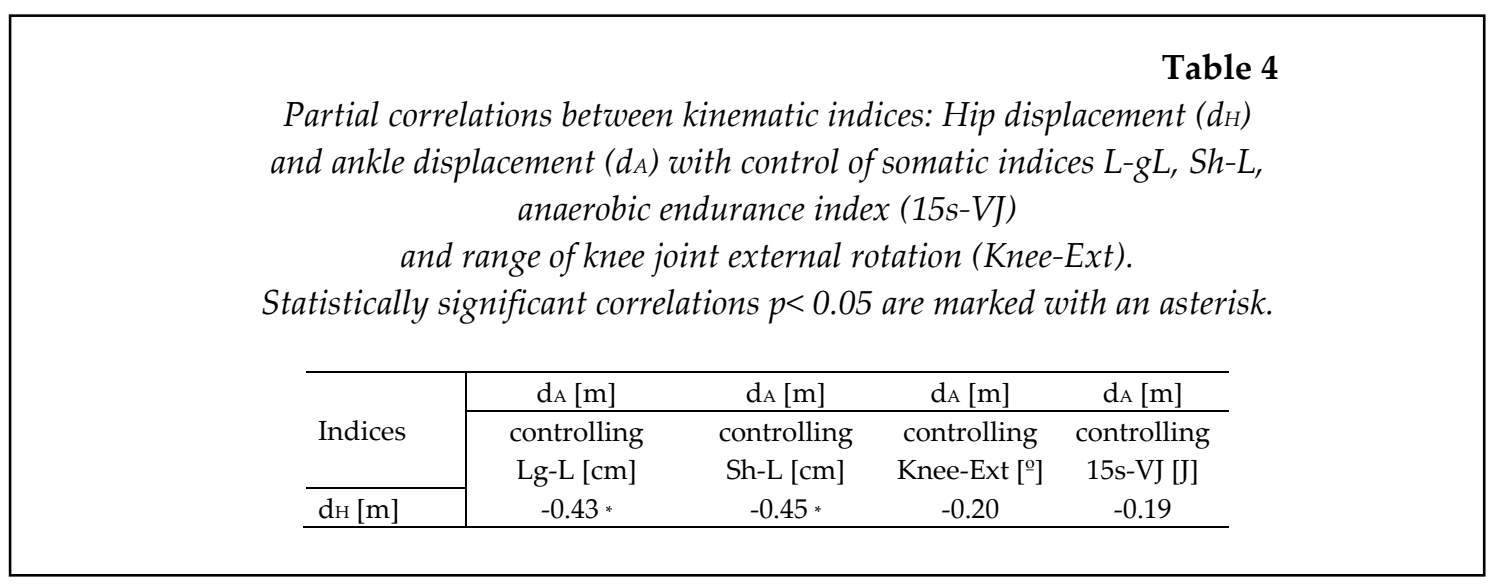

\section{Discussion}

The kinematic analysis of breaststroke kicks in our study may support observations of swimming specialists regarding the fact that a swimmer's talent is recognised by the so-called feeling of the water, which is manifested by precise and strong propulsive movements without excessive extremities slip-sink through the water. In our measurements, hip displacement $(\mathrm{dH})$ compatible with horizontal body displacement was not positively related to ankle joint displacement $\left(\mathrm{d}_{\mathrm{A}}\right)$ opposite to body displacement. Moreover, it was stated that these dependencies, $d_{H}$ to foot slip $\left(d_{A}\right)$ with control of lower extremity length indices $\mathrm{Lg}-\mathrm{L}$ and $\mathrm{Sh}-\mathrm{L}$, are negatively correlated.

Vorontsov and Rumyantsev (2000) in their literature review stated that leg actions are able to create greater hydrodynamic forces than arm actions for the following reasons: legs possess significantly greater propelling surface area, and the muscle groups of the legs are significantly stronger than those of the arms. However, in 
swimming, these features play a minor role in creating effective propulsive forces (Vorontsov and Rumyantsev, 2000). The results of our research support these statements.

In the present study, swimming speed using breaststroke kicks depended primarily on the anaerobic endurance index $(15 \mathrm{~s}-\mathrm{VJ})$. In addition to the $15 \mathrm{~s}-\mathrm{VJ}$, the knee external rotation (Knee-Ext) and the swimming technique index KL had a direct impact on swimming speed.

To generate an above-average propulsive force in multidimensional breaststroke kicks, besides muscular power Falk et al. (2004), Platanou (2005), it is vital to have a wide range of movement in each of the lower extremity joints. In the research of Jagomägi and Jürimäe (2005), external knee rotation had more of an influence on swimming speed in a $100 \mathrm{~m}$ breaststroke swim using a kickboard than did external hip rotation or ankle supination. All these dependencies on the average level were statistically significant in the group of female swimmers. Jagomägi and Jürimäe (2005) stated that the more flexible the ankles and knees are, the easier it is to keep the kick narrow and kick back rather than out. In contrast, the entire hip and ankle joint range of movement available in the breaststroke was not used by the most skilled swimmers in the Kippenhan's (2002) observations. However, they noted that the more skilled the subjects were, the greater the external knee rotation during the breaststroke kick, external knee rotation towards the end of the downsweep in particular. According to them, increasing the external knee rotation during this phase was essential for maintaining a more propulsive foot orientation for as long as possible.

In research on breaststroke kicks conducted by Matheson et al. (2011) in female NCAA Division I swimmers, four different approaches were observed, based on variations of movement of lower extremity segments. The authors present examples: two participants predominantly used a thigh and foot approach. Three participants used mainly adduction with most of the contribution coming from the thigh shank. Three others performed the kick from the knee down, using mainly shank and foot velocities, while four participants displayed a more distributed sequence by using significant contributions to velocity from all three segments.

\section{Conclusions}

The present research and measurements of selected body properties, physical endurance testing and kinematic movement analysis may help in precise detection of an athlete's talent for the breaststroke. The observed dependencies of anaerobic endurance (15s-VJ), kick length, as well as the knee joint movement range in the lateral plane on speed with regard to the breaststroke kicks form an important conclusion which may have an impact on swimming technique shaping as well as swimmers body abilities implicating speed and movement trajectory of the legs in breaststroke. Furthermore, our measurements of hip displacement $(\mathrm{dH})$ compatible with horizontal body displacement which was not positively related to foot slip $\left(\mathrm{d}_{\mathrm{A}}\right)$ may be considered helpful in the assessment of breaststroke kick efficiency.

\section{References}

Bixler BS. Resistance and propulsion. In Stager JM and Tanner DA. (eds.): Swimming. Handbook of Sports Medicine and Science. Blackwell Science Ltd. Massachusetts. 2005; 96

Falk B, Lidor R, Lander Y, Lang B. Talent identification and early development of elite water-polo players: a 2-year follow-up study. J Sport Sci, 2004; 22, 347-355

Gerard ES, Caiozzo VJ, Rubin BD, Prietto CA, Davidson DM. Skeletal muscle profiles among elite long, middle, and short distance swimmers. Am J Sports Med, 1986; 14: 77-82

Jagomägi G, Jürimäe T. The influence of anthropometrical and flexibility parameters on the results of breaststroke swimming. Anthropologischer Anzeiger, 2005; 2: 213-219

Kippenhan BC. Influence of lower extremity joint motions of the effectiveness of the kick in breaststroke swimming. In J.R. Blackwell \& R.H. Sanders (eds.), Proceedings of swim sessions: XIX International Symposium on Biomechanics in Sports 2001; 48-52. San Francisco, CA: University of San Francisco 
Kippenhan CB. Lower-extremity joint angles used during the breaststroke whip kick and the influence of flexibility on the effectiveness of the kick. ISBS - Conference Proceedings Archive, XX International Symposium on Biomechanics in Sports, ISSN 1999-4168. International Society of Biomechanics in Sports, University of Konstanz 2002; 31-34

Leblanc H, Seifert L, Chollet D. Arm-leg coordination in recreational and competitive breaststroke swimmers. Journal of Science and Medicine in Sport, 2009; 12: 352-356

Maglisho EW. Swimming fastest. Human Kinetics. Leeds. 2003; 229

Mason BR, Patton SG, Newton AP. Propulsion in breaststroke swimming. In W.E. Morrison (eds.), Proceedings of the VII International Symposium on Biomechanics in Sport, 1989; 257-267. Melbourne, Australia: Footscray Institute of Technology

Matheson E, Hwang Y, Romack J, Whiting W, Vrongistions K. A kinematic analysis of the breaststroke kick. Portuguese Journal of Sport Sciences, 2011; 11: Suppl. 2

Platanou T. On-water and dryland vertical jump in water polo players. J Sports Med Phys Fit, 2005; 45: 26-31

Schleihauf R. Kinematic Analysis Software (Version 5.91) Biomechanics Software: San Francisco State University 2006

Vorontsov AR., Rumyantsev VA. Resistive forces in swimming. In: Zatsiorsky V. (eds.): The Encyclopaedia of Sports Medicine. Blackwell Science Ltd. London. 2000; 224

Yeater RA, Martin RB, White MK, Gilson KH. Tethered swimming forces in the crawl, breast and back strokes and their relationship to competitive performance. Journal of Biomechanics, 1980; 14: 527-537

\section{Corresponding author \\ Marek Strzała}

Al. Jana Pawła II 78,

31-571 Kraków,

Phone: 0048 126831102,

E-mail: marek.strzala@awf.krakow.pl, marekstrzala@o2.pl 